\title{
Effects of Complex Symmetry-breakings on Alpha Particle Power Loads on First Wall Structures and Equilibrium in ITER
}

\author{
K. Shinohara 1), T. Kurki-Suonio 2), D. Spong 3), O. Asunta 2), K. Tani 4), \\ E. Strumberger 5), S. Briguglio 6), T. Koskela 2), G. Vlad 6), S. Günter 5), G. Kramer 7), \\ S. Putvinski 8), K. Hamamatsu 1), ITPA Topical Group on Energetic Particles
}

1) Japan Atomic Energy Agency, Naka, Ibaraki, Japan

2) Aalto university, Assn. Euratom-Tekes, P.O. Box 14100, FIN-00076 Aalto, Finland

3) Oak Ridge National Laboratory, Fusion Energy Theory Group, Oak Ridge, USA

4) Nippon Advanced Technology Co., Ltd., Naka Office, Naka, Ibaraki, Japan

5) Max-Planck-Institut-für-Plasmaphysik, EURATOM-Association, Garching, Germany

6) ENEA, Frascati, Italy

7) Princeton Plasma Physics Laboratory, Princeton University, USA

8) ITER Organization, Route de Vinon sur Verdon, F-13115 St Paul lez Durance, France

E-mail contact of main author: shinohara.koji@jaea.go.jp

\begin{abstract}
Within the ITPA Topical Group on Energetic Particles, we have investigated the impact that various mechanisms breaking the tokamak axisymmetry can have on the fusion alpha particle confinement in ITER as well as on the wall power loads due to these alphas. In addition to the well known TF ripple, the 3D effect due to ferromagnetic materials (in ferritic inserts, FI, and test blanket modules, TBM) and ELM mitigation coils are included in these mechanisms. ITER Scenario-4 was chosen since, due to its lower plasma current, it is more vulnerable for various off-normal features. First, the validity of using a $2 \mathrm{D}$ equilibrium was investigated: a $3 \mathrm{D}$ equilibrium was reconstructed using the VMEC code, and it was verified that no 3D equilibrium reconstruction is needed but it is sufficient to add the vacuum field perturbations onto an axisymmetric equilibrium. Then the alpha particle confinement was studied using three independent codes, ASCOT, DELTA5D, and F3D OFMC, all of which assume MHD quiescent background plasma and no anomalous diffusion. All the codes gave a loss power fraction of about $0.2 \%$. The distribution of the peak power load was found to depend on the first wall shape. We also made the first attempt to accommodate the effect of fast ion related MHD on the wall loads in ITER using the HMGC and ASCOT codes. The power flux to the wall was found to increase due to the redistribution of fast ion by the MHD activity. Furthermore, the effect of the ELM mitigation field on the fast ion confinement was addressed by simulating NBI ions with the F3D OFMC code. The loss power fraction of NBI ions was found to increase from $0.3 \%$ without the ELM mitigation field to $4-5 \%$ with the ELM mitigation field.
\end{abstract}

\section{PACS:}

\section{Introduction}

The new physics introduced by ITER operation, of which there is very little prior experience, is related to the very energetic $(3.5 \mathrm{MeV})$ alpha particles produced in large quantities in fusion reactions. These particles not only constitute a massive energy source inside the plasma, but they also present a potential hazard to the material structures that provide the containment of the burning plasma. Analysing the alpha particle behaviour is one of most important activities in our group, the ITPA Topical Group on Energetic Particles.

In most theoretical analyses, the tokamak magnetic field is assumed axisymmetric. However, in reality, this symmetry is broken. As is well known, the finite number and limited toroidal extent of the Toroidal Field (TF) coils cause a periodic variation of the toroidal field called the magnetic ripple. This ripple can provide a significant channel for fast particle leakage, leading to very localized power loads on the plasma-facing components. Because of this, 
Ferromagnetic Inserts (FIs) will be embedded in the double wall structure of the ITER vacuum vessel in order to reduce the ripple[1,[2]. In ITER, the toroidal field perturbations are further locally enhanced by the presence of discrete ferromagnetic structures, the Test Blanket Modules (TBM). Thus, there are complex symmetry-breakings in ITER. It seems that breaking the toroidal symmetry introduces islands to the magnetic field structure. Furthermore, the resulting 3D nature of the plasma equilibrium has been suspected to provide an additional mechanism for the fusion alphas to leak out at unacceptable level [3]. It is not yet theoretically understood how superimposing the periodic ripple and a local perturbation affects the fast ion confinement, and concerns have been voiced that the combined effect might introduce new, unexpected channelling mechanisms for fast ions.

Preliminary studies on alpha particle confinement have been carried out by using a variety of 5D Monte Carlo guiding-center codes (3D in the real space, and $\mathrm{v}_{\text {para }}$ and $\mathrm{v}_{\text {perp }}$ in velocity space for the guiding center): OFMC[4, [7], HYBRID[5, [6, [7], ASCOT[8, [3], DELTA5D [9], and an upgraded version of OFMC, F3D OFMC code[12, [13, [14]. In fact, the results of Ref [7] by OFMC and HYBRID codes revealed the importance of the reduction of TF ripple in ITER, and the installation of FI was proposed. Also the full orbit-following code, SPIRAL[10], has been used for confinement studies, while preliminary results for the power loads on ITER wall structures were obtained using ASCOT[3].

These simulations were carried out using data that has later been changed (the wall structure and geometry of FIs) or found deficient (the 3D vacuum magnetic field corresponding to the situation with TBMs). In this work, the wall power loads due to fusion-born alpha particles are re-studied for a variety of cases addressing issues such as different wall configurations, proper inclusion of the TBM effect on the vacuum magnetic field, and the possible corrections to $3 \mathrm{D}$ equilibrium introduced by the ferromagnetic materials. In the past it was customary to calculate the effect of the ferromagnetic materials on the vacuum field only. The equilibrium issue is accomplished using the full free-boundary 3D equilibrium code, VMEC [11]. We also study the effect of the ELM mitigation coils on fast ion confinement and make an attempt to assess the effect of fast-ion related MHD on alpha particle confinement and wall loads.

The paper is organized as follows. Section 2 presents a careful analysis of the 3D magnetic field using VMEC and FEMAG codes. In Sec. 3, the confinement of fusion alphas is investigated and the power load on a number of different wall shapes is evaluated using three independent codes: ASCOT, DELTA5D and F3D OFMC. In this Section, also the impact that the ELM mitigation coils and fast-ion related MHD might have on alpha confinement is investigated. Finally, in Section 4, we summarize our results and propose future work.

\section{Re-construction of the 3D magnetic field}

The orbits of collisionless ions are entirely determined by the 3D magnetic field. Therefore we need to very accurately model the 3D magnetic field that includes the poloidal field by the plasma current and poloidal field coils. We first calculate the 3D vacuum magnetic field, considering the effect of ferromagnetic material, and then we investigate the effect of 3D vacuum magnetic field on the plasma equilibrium. Here, by "vacuum magnetic field" we refer to the magnetic field which does not include the poloidal field generated by the plasma and poloidal field coils. 
The 3D vacuum magnetic field was calculated by using the FEMAG code [12] to properly include the TBM effect. FEMAG calculates the effect on the total field including the poloidal field due to the plasma current as well as the vacuum field. The FEMAG code models the ferromagnetic material as thin plates with magnetic charge on the edges as depicted in Fig. 1. The magnetic charge is distributed according to the direction of the external magnetic field and its strength is determined by the saturated magnetization of the ferromagnetic material. When the external magnetic field is larger than $1 \mathrm{~T}$, which is typical in most tokamak devices, full saturation of the ferritic steel is achieved. The external field includes not only the toroidal field but also the poloidal field due to the plasma current and poloidal coils. The input parameters, such as the geometry data of FIs, are the same as in Ref. [14] (Sec. 2.3 and 3.1), and correspond to the case where FI [15] and Helium-Cooled Lithium-Lead (HCLL) TBM $[16,[17]$ are installed as shown in Fig. 2. The FIs are installed keeping the 18-fold symmetry (20-degree periodicity) in the toroidal direction without considering the irregular port section for the NB injectors. The toroidal variation of the toroidal field is plotted in Fig. 3. In the three TBM sections, at around 30,310, 350 degrees, the ripple amplitude is seen to be enhanced by the TBM-induced field bump.

Ferromagnetic material affects the strength of the magnetic field and slightly changes its direction outside the ferromagnetic regions. Therefore, in order to properly include the effect of FIs and TBMs on the ITER magnetic field, it might not be sufficient to calculate the effect on the vacuum field only. We need to investigate the effect on the plasma equilibrium. Usually the plasma equilibrium is calculated in $2 \mathrm{D}$, assuming axisymmetry. However, the 3D vacuum magnetic field/perturbation might affect the axisymmetric current. Furthermore, the resultant 3D equilibrium might induce additional symmetry-breaking. This mechanism might be enhanced through the modified plasma diamagnetic and Pfirsch-Schlüter currents when finite $\beta$ effects are included. In order to assess the significance for an ITER plasma, a 3D equilibrium was calculated using the full free-boundary 3D equilibrium code, VMEC [11]. The vacuum magnetic field, the total toroidal flux, as well as the pressure and safety factor are input data for the code. VMEC then calculates the 3D equilibrium by minimizing the total energy of a plasma confined to a toroidal domain, assuming nested flux surfaces.

ITER plasma corresponding to Scenario-4 operation was chosen as the subject of this study. Scenario 4 is a steady state 9MA scenario with a weak negative magnetic shear, a highly shaped plasma, and a volume-averaged plasma beta of $\langle\beta\rangle=2.24 \%$, producing about $300 \mathrm{MW}$ of fusion power with $\mathrm{Q}=5$ for $3000 \mathrm{~s}$ [18]. Even though the power production in Scenario-4 is smaller than in Scenario-2, due to its lower plasma current the fast ion orbits are wider and, thus, fast ions in Scenario-4 plasma are more vulnerable to off-normal field configurations. Here, Scenario- 2 is an inductive 15 MA scenario with a full bore plasma producing $400 \mathrm{MW}$ of fusion power with a fusion gain $\mathrm{Q}=10$ for $400 \mathrm{~s}$ [18]. The 2D equilibrium, q-profile, density profile, and temperature profiles for Scenario-4 are plotted in Fig. 4. The field aberration is compared between a full $3 \mathrm{D}$ equilibrium reconstruction and the standard case, where an axisymmetric equilibrium is added to the vacuum fields. Two approaches were used to constrain the current distribution. In the first approach, the q-profile was given. In the second approach, the current profile itself was given. The field aberration/ripple $\delta$ is defined as $\delta=\left(B_{\max }-B_{\min }\right) /\left(B_{\max }+B_{\min }\right)$, and, thus, in the absence of any TBM field bump it reduces to the regular toroidal ripple.

First, with a given q-profile, the effect of the pure, unmitigated TF ripple (no FI or TBM) was investigated. Also the $\beta$-dependence of the effect was studied [20]. The unmitigated TF ripple reaches $1 \%$ at the surface of the plasma [14]. It was found that the effect of the TF ripple 
increased with increasing $\beta$-value, but the effect was negligibly small. The maximum increase in the ripple amplitude was about $0.05 \%$ even for the case of $\langle\beta\rangle=4.67 \%$ (Fig. 2 in Ref. [20]). That is, the maximum relative difference of the ripple amplitude was about $5 \%$. The same study was then repeated for the non-axisymmetric vacuum field including the FI and TBM contributions. However, here the $\beta$-scan was limited: for $\beta$-values higher than $\langle\beta\rangle=$ $2.24 \%$, the $n=1$ perturbation increased. Stability analysis with the CASTROR_FLOW code [21] revealed that higher- $\beta$ equilibria are strongly unstable with respect to $n=1$ ideal kink modes. Even so, the difference between 3D and 2D (axisymmetric equilibrium + vacuum perturbation field) equilibria was negligibly small as shown in Fig. 5, where the radial difference, $d R$, of flux surfaces (field lines) at $\mathrm{Z} \sim 0.5 \mathrm{~m}$ is compared. In Fig. 5(a), the flux surfaces computed with the NEMEC code from the VMEC output are shown. NEMEC assumes nested flux surfaces, so it doesn't produce islands. Fig. 5(b) depicts field line traces at positions corresponding to that in Fig. 5(a). In these figures, the red lines (dots) correspond to flux surfaces (field lines) for the toroidal angle of 30 degree, where a TBM is located. The black (dots) correspond to flux surfaces (field lines) for the toroidal angle of 20 degree, which corresponds to the location of a TF coil. Fig. 5(c) shows the radial variation of the radial difference, $d R$, of flux surfaces (field lines) at Z 0.5m, and Fig. 5(d) shows the ratio of the radial differences of the $2 \mathrm{D}$ to $3 \mathrm{D}$ equilibria, namely the ratio of the values of " $\mathrm{x}$ " makers to "o" markers in Fig. 5(c). The radial difference is $\sim 0.1 \%$ of the major radius for both $3 \mathrm{D}$ and $2 \mathrm{D}$ equilibria, and the difference of these two methods is less than $\sim 0.01 \%$ of the major radius.

The VMEC analysis was also carried out using the second approach, where the current density was given. The ripple distribution, shown in Fig. 6, was again found small. The VMEC analysis also showed that finite- $\beta$ effects could modify ripple levels through the induced plasma diamagnetic and Pfirsch-Schlüter currents, see Fig. 7, where the result for $\langle\beta\rangle=2.6 \%$, rather than $\langle\beta>=2.24 \%$ (the standard value), is plotted as a test case to study the sensitivity to beta.

From these VMEC analyses, it can be concluded that no 3D equilibrium reconstruction is needed for the numerical production of the magnetic field but that it is sufficient to add the vacuum field perturbations onto an axisymmetric equilibrium for the reconstruction of the equilibrium in the ITER.

In this analysis, we noticed that the ripple area change when we define magnetic field at the fixed poloidal flux and poloidal angle instead of the fixed radial and vertical position, $(\mathrm{R}, \mathrm{Z})$. The contour map of ripple for the fixed poloidal flux and poloidal angle is shown in Fig. 8 . The ripple area somewhat extended to the inner region of plasma because the poloidal flux is moving in the radial direction in the $3 \mathrm{D}$ equilibrium. This might suggest the thermal plasma, which is considered to be a function of poloidal flux, can be affected by 3D magnetic perturbation in the inner region.

Also the structure of the Alfvén continuum was investigated for the 3D equilibrium. Fig. 9 shows the $\mathrm{n}=1$ mode family continuum for ITER with TF ripple, FIs and TBMs. It was found that the 3D field aberrations produces new ripple gaps. Though its radial structure is small, it might affect the damping mechanism of the Alfvén eigenmodes.

\section{Confinement of fast ions and power load on the first wall}

\subsection{Benchmark and the effect of different wall shapes}


Under the framework of the ITPA Topical Group on Energetic Particles, we decided to simulate the fast ion confinement and power load on the first wall using two independent codes: ASCOT and F3D OFMC.

The comparison between the experimental data and the simulation were carried out in the present-day tokamaks, independently. The comparisons with the data of the triton surface deposition and the data of the neutral particle analyzer were carried out in the ASDEX-U for ASCOT [22, [23]. The comparisons with energetic deuteron confinement estimated by the neutron emission decay and the wall load estimated by the infrared TV camera measurement were carried out in the JT-60U [24], and the comparisons of the wall load estimated by the infrared TV camera measurement were carried out in the JFT-2M under the environment with ferritic inserts [12] for F3D OFMC. Recently, the comparison with the data from the thermocouple measurement were carried out in the TBM mock-up coil experiments in DIII-D for both of ASCOT, F3D OFMC, and SPIRAL [25, [25]. In these comparisons, the simulation is consistent with the experimental data, considering the error in the experiments and the simulations. We are planned to have a better comparison in the TBM mock-up coil experiments in DIII-D in the near future.

It was assured that both codes had identical input data corresponding to the ITER Scenario-4 operation described above. The $2 \mathrm{D}$ equilibrium and profiles of plasma parameters are shown in Fig. 4. The 3D vacuum magnetic field, including the TF ripple, the correction field by the FIs and the perturbation by TBMs, was calculated by FEMAG. The confinement of the fusion alphas was simulated assuming only neoclassical transport via Coulomb collisions with a stationary, Maxwellian background plasma, i.e. neglecting any MHD activity or turbulent transport. The alpha particles are followed until their trajectory reaches the wall or their energy drops below $100 \mathrm{keV}$ for ASCOT and below $32 \mathrm{keV}$ for OFMC. As a benchmark study, an axisymmetric first wall, shown in Fig. 4 (a), was applied.

The loss power fraction and the loss particle fraction, calculated by ASCOT and F3D OFMC, was found to be about $0.2 \%$ and about $1 \%$, respectively. The power load distribution is plotted in Fig. 10. The energy range which contributes to the power load is less than $1 \mathrm{MeV}$ in this particular case. The peak power load is less than $50 \mathrm{~kW} / \mathrm{m}^{2}$. This value is well below the critical level of $\sim 1 \mathrm{MW} / \mathrm{m}^{2}$ for this region. Thus, this combination of the FI, TBM, wall shape, and plasma shape is acceptable from the material tolerance point of view. It should be noted that the dominant region of power load distribution is not necessarily around the outer midplane: in Fig. 10 the belt of large power flux is found at about 50 degrees below the poloidal angle. This is in contrast to previous works where only little power was observed in this region for the same 3D vacuum magnetic field but somewhat different wall shape [14], and for somewhat different magnetic field and somewhat different wall shape [3]. Thus the power load clearly depends on the first wall shape.

We then decided to investigate the sensitivity of the wall distribution on the 2D shape of the wall. Fig. 11 displays the different (axisymmetric) wall shapes used in F3D OFMC simulations. In the earlier work, the wall was the so-called limiter-like wall, labeled as $\mathrm{W}_{\text {old }}$ in Fig. 11(b), and it was closer to the plasmas than the wall used in the present study (labeled "W-benchmark"). The intermediate wall shapes, which were artificially prepared for this wall shape scan, are labeled as "W\#1" and "W\#2". In Fig. 11, power load distribution resulting from F3D OFMC simulations are shown for the different wall shapes. As the wall moves away from the plasma, the power load around the outer midplane diminishes, while the load on the lower position increases. Fig. 12 shows the relation between the wall and the magnetic 
field line by poloidal flux contour lines. The position where the peak heat load is observed corresponds to the position where the field lines first intersect the wall. The corresponding field line is also indicated in Fig. 11(a) and (b) by a green line. This is qualitatively reasonable though we need to note the orbit of fast ions differ from the field line.

ASCOT is capable of modeling 3D wall structures and was used to check the effect of limiter structures. The simulation was carried out with an earlier ITER design with two limiters located 180 degrees from each other. Fig. 13 shows the ASCOT results and indicates somewhat higher peak heat flux on the limiters, though the values are still acceptable. A more recent analysis of the 3D fast ion power load on the recent design of ITER first wall with 18 limiters presents a different power load pattern.

The effect of wall shape was also carried out with DELTA5D. Here, the wall shape in DELTA5D is proportional to the plasma shape. The dependence of the energy distribution of lost ions on the distance between the plasma and the wall is shown in Fig. 14. Interestingly, we can find a resonant behavior of the energy distribution around $1.5 \mathrm{MeV}$, when the wall is close to the plasma.

These results from the wall shape study indicate the importance of re-evaluating the power loads following updates in wall shape geometry and plasma equilibrium.

\subsection{Effect of MHD on the heat load}

As mentioned earlier, all the confinement and wall load simulations were carried out assuming purely neoclassical transport. However, it is clear that plasmas with a large population of energetic ions might exhibit various fast ion -related MHD phenomena such as Alfvén eigenmodes. ITER, with its high fusion yield, will not be an exception. This MHD activity will act back on the energetic ions possibly transporting them towards the plasma edge, where the TF ripple and TBM field bump provide an efficient mechanism for moving ions to the plasma-facing components. Therefore we wanted to find a way to simulate the wall power load not only from the slowing-down distribution of the fusion alphas but also from a distribution that has been affected by the relevant MHD.

The Hybrid MHD-Gyrokinetic Code (HMGC) [27] can calculate the 4D (e.g. ( $\left.\rho, \theta, \mathrm{v}_{\text {perp }}, \mathrm{V}_{\text {para }}\right)$ ) alpha particle density in the presence of Alfvén modes. In the HMGC simulations, it was found that the $n=2$ Alfvén modes that peak around the minimum-q position can appear. The interaction of these $n=2$ Alfvén modes on the fusion alphas in the ITER Scenario 4 plasma was evaluated [28]. The resulting alpha distribution was then used in ASCOT as input, and the simulation results were compared to a simulation in the absence of Alfvén eigenmodes. Naturally, the proper way of addressing the effect of MHD activity on fast ion distribution would be to follow the test particles in the time-dependent perturbed field due to the MHD activity because wave-particle interaction is an important mechanism in this kind of transport. However, the wave-particle interaction itself, simulated in nonlinear MHD codes such as HMGC and MEGA [29], needs enormous computing resources and a comprehensive approach remains a future challenge. Therefore we had to settle for a first-order comparison of results that are obtained simply using different input profiles for ASCOT. 
In ASCOT, normally the particle input is a source rate profile of either the fusion born alpha particles or NBI-ions. The steady-state contribution to the wall structures in then obtained by integrating over the contribution of these particles during their life time, which is dictated either by their slowing down time or the time it takes them to intersect a material structure. In this analysis, however, only a steady state phase space density profile was available from HMGC as input to ASCOT. The meaningful wall simulation should then consider only prompt and almost prompt losses since there is no way of keeping the profile stationary. Therefore the wall load was evaluated following the orbits of test particles for only $100 \mu \mathrm{s}$. This is sufficiently long to account for the prompt losses of alphas redistributed by Alfvén activity but too short to account for ripple-enhanced diffusion.

The wall power loads obtained from the ASCOT simulations using the two different initial alpha distributions are shown in Fig. 15. The two different alpha distributions used in ASCOT wall power load simulations are dubbed "initial" and "saturated". The name "initial" refers to the slowing-down distribution at the time when MHD activity only starts to evolve, while the name "saturated" corresponds to the time when MHD activity is saturated in HMGC. The population of energetic alpha particles is increased at the outer region due to the redistribution by the Alfvén modes. In $100 \mu \mathrm{s}$, the loss energy that, in the case of "initial" distribution, was about $15 \mathrm{~kJ}$, was increased to about $50 \mathrm{~kJ}$ in the case of "saturated" distribution. The toroidally averaged wall load for these two cases is shown in Fig. 16. The heat flux to the wall is seen to significantly increase particularly at the outer midplane. The peak heat flux is increased by up to an order of magnitude in the most vulnerable regions.

\subsection{Effect of magnetic field by ELM mitigation coils}

In order to protect and guarantee a reasonable lifetime for the divertor plates in ITER, the type I ELMs have to be mitigated at least. One way of accomplishing this is via ELM mitigation coils that change the magnetic field structure in the pedestal region thus providing yet another symmetry-breaking mechanism for the ITER magnetic field. The design of ELM mitigation coils is still on going in ITER.

We investigated the effect of ELM mitigation field on the fast ion confinement based on the 2009 design [30]. The coil system consists of 27 coil sets. The 9 sets of coils are distributed at every 40 degrees in the toroidal direction. Each toroidal set consists of three coils placed on the upper, equatorial, lower position on the low field side. The coil current is configured to produce an $n=4$ perturbation, and the phase of the coil current of the upper and lower coils is shifted by -12.4 and -3.4 degrees, respectively, compared to the equatorial coil thus providing a match with Scenario 4 operation. The field due to the ELM mitigation coils, shown in Fig. 17(a), is added to the 3D field described above, which includes the TF ripple as well as the perturbation by FIs and TBMs. That is, we add the "vacuum" ELM mitigation field to the 3D vacuum magnetic field. Thus, the shielding effect of plasmas on field penetration is not considered. An appropriate model of the shielding effect is still missing and the construction of the model is out of the scope of this paper. The resulting field variation in the toroidal direction is plotted in Fig. 17(b).

The effect of the ELM mitigation field on fast ion confinement was investigated using the F3D OFMC code. The loss power fraction of alpha particles was found to slightly increase from $0.2 \%$ without the ELM mitigation field to $0.35 \%$ with the ELM mitigation field. On the other hand, the loss power fraction of fast NBI deuterium ions was found to increase from $0.3 \%$ without the ELM mitigation field to $4-5 \%$ with the ELM mitigation field. This level of 
loss power fraction might not be negligible. It is thus necessary to continue simulating the effect of the ELM mitigation field in the updated designs and other background plasmas.

\section{Summary}

Within the activity of ITPA Topical Group on Energetic Particles, we have investigated the 3D effect of ferromagnetic materials and ELM mitigation coils as well as a TF ripple, i.e. complex symmetry-breaking effects in ITER.

To start with, we investigated the effect of the 3D vacuum magnetic field on the equilibrium for Scenario 4. With a self-consistent 3D equilibrium reconstruction using the VMEC code, it was concluded that no 3D equilibrium reconstruction is needed for the numerical production of the magnetic field but that it is sufficient to add the vacuum field perturbations onto an axisymmetric equilibrium on ITER even with a TBM perturbation which is the largest magnetic perturbation.

Using identical input data for both magnetic background and particle initialization, the evaluation of the alpha particle confinement was carried out by using ASCOT and F3D OFMC codes. As the result, the loss power fraction is about $0.2 \%$ and is comparable with ASCOT and F3D OFMC.

Sensitivity of the peak heat load on the first wall shape was observed. In the analysis of different 2D wall shapes using F3D OFMC, the peak heat load was not always found at the TBM position when the wall shape is changed only around the midplane region. ASCOT simulations with a 3D wall further indicated that the limiter structures, locally placed in the toroidal direction, received about an order of magnitude larger peak heat flux. DELTA5D showed the dependence of the energy distribution of lost ions on the distance between the plasma and the wall. These results for the wall shape dependence indicate the importance of re-evaluating the power loads following updates in the wall shape and equilibrium.

An attempt to accommodate the effect of fast ion related MHD activity on the wall load in ITER was carried out using the ASCOT code. In this attempt, the heat load was compared for two fast ion profiles calculated by the HMGC code: one at the time when MHD activity only starts to evolve, and the other for saturated MHD activity. The peak power flux to the wall due to direct losses was found to increase by an order of magnitude. Thus future, more comprehensive studies on the combined effect of core MHD and edge magnetic asymmetries are called for.

We also investigated the effect of ELM mitigation coils on the fast ion confinement based on the 2009 design. Adding the "vacuum" ELM mitigation field to the 3D vacuum magnetic field, the loss power fraction of alpha particles was found to slightly increase from $0.2 \%$ without the ELM mitigation field to $0.35 \%$ with the ELM mitigation field. On the other hand, the loss power fraction of NBI-generated fast ions increased from $0.3 \%$ without the ELM mitigation field to 4-5\% with the ELM mitigation field. It will thus be important to re-asses the effect of ELM mitigation field on fast ion confinement when the design of ELM mitigation coils is updated.

The ITPA Topical Group on Energetic Particles will continue to contribute to the ITER design from at least the following aspects: following up on changes in wall shape, plasma shape, and ELM mitigation coil design, we shall re-evaluate the wall power loads. We shall 
also work on incorporating various MHD effects, error fields, and turbulent transport to get more realistic numerical estimates for the wall loads and fast ion confinement.

The views and opinions expressed herein do not necessarily reflect those of the ITER Organization

\section{Acknowledgments}

We would like to thank to Drs V. Mukovatov, T. Oikawa, S. Konovalov, J. Snipes, Y. Gribov, $\mathrm{V}$ Chuyanov for their encouraging our activity and providing us useful information. Part of this work was supported by the Academy of Finland Project 121371. The supercomputing resources of CSC - IT center for science were utilized in the studies for the ASCOT code.

\section{Reference}

[1] Shimada M., et al., Progress in the ITER Physics Basis, 'Chapter 1: Overview and summary', Nucl. Fusion 47 (2007) S1

[2] Fasoli A., et al., Progress in the ITER Physics Basis, 'Chapter 5: Physics of energetic ions', Nucl. Fusion 47 (2007) S264

[3] Kurki-Suonio T., et.al., Nucl. Fusion 49 (2009) 095001

[4] Tani K., et. al., J. Phys. Soc. Jpn., 50 (1981) 1726

[5] Konovalov S.V., et al. "Analysis of high energy ion ripple loss in the up-down asymmetric configuration by OFMC plus mapping hybrid code", JAERI-Research 94-033 (1994)

[6] Konovalov S.V., "Alpha particle ripple loss in S4 and S2 ITER reference scenarios", ITER Physics Design Task 2, RRC “Kurchatov Institute”, July 20, 2001

[7] Tobita K., Nakayama T., Konovalov S. V., and Sato M., Plasma Phys. Control. Fusion 45 (2003) 133

[8] Heikkinen J. A. and Sipilā, Phys. Plasmas 2 (1995) 3724

[9] Spong D. A., Hirshman S. P., Whitson J. C., Plasma Physics Reports, 23 (1997) (page 483 , sect. 3 )

[10] Kramer G., et.al. "First wall heat loads and fluences from fusion-born alpha particles in ITER", 2nd meeting of the Energetic particle Topical Group, Daejeon, Korea, (2009)

(http://itpa.ipp.mpg.de/energetic_particles/meeting_2009_02/)

[11] Hirshman S.P. and Lee D.K. Comput. Phys. Commun. 39 (1986) 161

[12] Shinohara K., et. al., Nucl. Fusion 43 (2003) 586

[13] Shinohara K., et.al. , Nucl. Fusion 47 (2007) 997

[14] Shinohara K., et.al., Fusion Engineering and Design 84 (2009) 24

[15] Private communication, Morimoto M., Mitsubishi heavy industries, LTD., Table 2 in "Segmentation of the ferromagnetic insert", 21-Aug-2007

[16] Rampal G., et. al., Fusion Engineering and Design 75-79 (2005) 917

[17] Private communication, Rampal G., CEA Saclay, France, " Simplified model of the HCLL TBM ferritic-martensitic (EUROFER structure)", HCLL TBM FM steel model_21Jun07.pdf

[18] Polevoi A.R., Medvedev S.Yu., Pustovitov V.D., et al., in Fusion Energy 2002 (Proc. 19th Int. Conf. Lyon, 2002) (Vienna: IAEA) CD-ROM file CT/P-08 and http://www.iaea.org/programmes/ripc/physics/fec2002/html/fec2002.htm

[19] Ivanov A.A., Medvedev S.Yu., "Comments on EQDSK Files for Reference ITER Scenarios 2 and 4 for Extended Area”, ITER D 27SVYS, 2007

[20] Strumberger E. et al., Nuclear Fusion $\mathbf{5 0}(2010) 025008$

[21] Strumberger E. et al Nucl. Fusion 45 (2005) 1156

[22] Kurki-Suonio T. , Hynönen V., Ahlgren T., et. al., Europhysics Letters 78 (2007) 65002. 
[23] Jämsä S., Kurki-Suonio T., Suttrop W., et.al. , 36th EPS Conference on Plasma Physics, Sofia, Bulgaria, June 29 - July 3, 2009; Europhysics conference abstracts, 2009. Vol. 33E, P1.148:1 - 4; http://epsppd.epfl.ch/Sofia/pdf/P1_148.pdf

[24] Tobita K. et al Nucl. Fusion 35 (1995) $158 \overline{5}$

[25] Kramer G., et.al. et al submitted to Nucl. Fusion

[26] Koskela T., Asunta O., Kramer G. J., et. al., "ASCOT modelling of fast ion losses in DIII-D TBM experiments", to appear in Europhysics Conference Abstracts, 2010.

[27] Briguglio S., Zonca F., and Vlad G., Phys. Plasmas 5, (1998) 3287

[28] Vlad G. et al., Nucl. Fusion 46 (2006) 1.

[29] Todo Y. and Sato T., Phys. Plasmas 5 (1998) 1321

[30] Private communication, M. J. Schaffer, General Atomics, U.S., INTERIUM REPORT ON IMPACT OF PROPOSED "MODIFIED BASELINE" AND "ALTERNATIVE VACUUM VESSEL” DESIGNS ON ITER ELM COILS, 26-Jun-2009 (2009) 


\section{Figure caption}

Fig. 1 A schematic diagram of magnetic charge model in the FEMAG code.

Fig. 2 The FI and TBM distribution modeled in this calculation

Fig. 3 Toroidal variation of the toroidal field strength of the vacuum magnetic field at $(R, Z)=(8.0 \mathrm{~m}$, $0.5 \mathrm{~m})(\mathrm{a})$, and the amplitude of the variation defined by $(\mathrm{Bt}-\langle\mathrm{Bt}\rangle) /\langle\mathrm{Bt}\rangle(\mathrm{b})$. Here, the sign of $\mathrm{Bt}$ is positive when, as viewed from above, the direction of $\mathrm{Bt}$ is counter-clockwise, and $\langle\mathrm{Bt}\rangle$ is a toroidal average.

Fig. 4 The 2D equilibrium with the first wall(a), the density (b), the electron (dashed line) and ion temperature (c), and the safety factor profile (d) for Scenario 4.

Fig. 5 The effect of the TBMs on the magnetic flux surfaces for 3D equilibrium calculations (a), and field lines for the 2D (axisymmetric equilibrium + vacuum perturbation field) case (b). The red lines (dots) correspond to flux surfaces (field lines) for the toroidal angle of 30 degrees, namely around a TBM section. The black (dots) correspond to flux surfaces (field lines) for the toroidal angle of 20 degrees, namely under a TF coil. (c) The radial difference, $d R$, of flux surfaces (field lines) at $Z \sim 0.5 \mathrm{~m}$ versus radial position of flux surfaces (field lines) at $Z \sim 0.5 \mathrm{~m}$ at toroidal angle of 20 degrees. The " $\mathrm{x}$ " marker is for 3D equilibrium calculations and "o" marker is for the $2 \mathrm{D}$ case, while (d) shows the ratio of the radial difference of $2 \mathrm{D}$ to $3 \mathrm{D}$ equilibrium.

Fig. 6 Ripple contours $(\delta)$ calculated on constant $(\mathrm{R}, \mathrm{Z})$ points (outer flux surface superimposed in blue) for: (a) vacuum fields. (b) VMEC finite- $\beta$ equilibrium fields for $\langle\beta\rangle=2.24 \%$. (c) absolute value of difference between (a) and (b).

Fig. 7 Plasma beta dependence of flux surface shapes and ripple amplitude. (a) Flux surface shapes for $<\beta>=1.5 \%$ (red), $<\beta>=2.6 \%$ (black). (b) Variation of maximum ripple within a flux surface vs. the normalized toroidal flux and $<\beta>$.

Fig. 8 Ripple contours calculated on constant $\Psi, \theta$ points using VMEC equilibrium fields with TBM's, ferritic inserts and TF coils, corresponding to Fig. 6 (b) for $\langle\beta\rangle=2.24 \%$. 
Fig. 9 The $\mathrm{n}=1$ mode family of Alfvén continua with TF ripple, FIs and TBMs. The TBM field is amplified by a factor of 5 in this figure to enhance the new gap.

Fig. 10 Heat load distribution for ASCOT (a) and F3D OFMC (b). The horizontal axis is the toroidal angle starting from TFC-1. TBMs are installed at around 30, 310, and 350 degrees. The vertical axis is the poloidal angle starting at the outer midplane as illustrated in (c).

Fig. 11 Toroidally averaged poloidal distributions of heat load for various wall shapes. (a) The shape of the plasma and the first wall, (b) a blow-up of figure (a), (c) poloidal heat load distribution for wall shape $\mathrm{W} \# 1$, (d) for $\mathrm{W} \# 2$, and (e) for W-benchmark.

Fig. 12 (a) The contour of the poloidal flux surfaces in Scenario 4 and the axisymmetric wall. (b) A blow-up of the region indicated in (a). The field lines indicated by green do not intersect the wall around the midplane but, rather, intersect the wall in the region circled in (b).

Fig. 13 (a) 3D wall model used in the ASCOT simulation with limiters. The model limiter structure is marked up, which protrudes $0.8 \mathrm{~cm}$ towards the plasma. (b) Poloidal cross section of the model wall at three toroidal position indicated in (a). (c) Expanded view around the outer midplane. The wall model is built of sectors that are symmetric except at the midplane ports. (d) Heat load calculated by ASCOT for the case with two limiters placed at 180 degrees apart. The poloidal angle starts at the outer midplane and increases in the counter-clock-wise direction when viewed from above.

Fig. 14 The dependence of the energy distribution of lost ions on the distance between a plasma and a wall.

Fig. 15 The initial distribution of alpha particles, obtained from the HMGC simulations, for the ASCOT wall load simulations. (a) The distribution corresponding to the time when $n=2$ Alfvén modes start to grow, and (b) the distribution corresponding to the saturated Alfvén modes. (c) and (d) give close-ups on the distribution near the plasma edge, $\psi>0.8$. The phase space densities in (a) and (b) are normalized to the maximum value of (a), $1.8 \times 10^{19}\left[\mathrm{~m}^{-3} \mathrm{eV}^{-1}\right]$, and the densities in (c) and (d) to the maximum value of (c), $3.0 \times 10^{17}\left[\mathrm{~m}^{-3} \mathrm{eV}^{-1}\right]$.

Fig. 16 Wall load for the "initial" distribution and for the "saturated" distribution. The poloidal angle starts at the outer midplane and increases in the counter-clock-wise direction when viewed from above.

Fig. 17 Toroidal variation of toroidal field strength of the vacuum magnetic field. (a) With the ELM mitigation coils alone. (b) With ELM mitigation field plus $3 \mathrm{D}$ vacuum magnetic field at $(\mathrm{R}, \mathrm{Z})=(8.2$ $\mathrm{m}, 0.6 \mathrm{~m}$ ). 


\section{Fig 1}

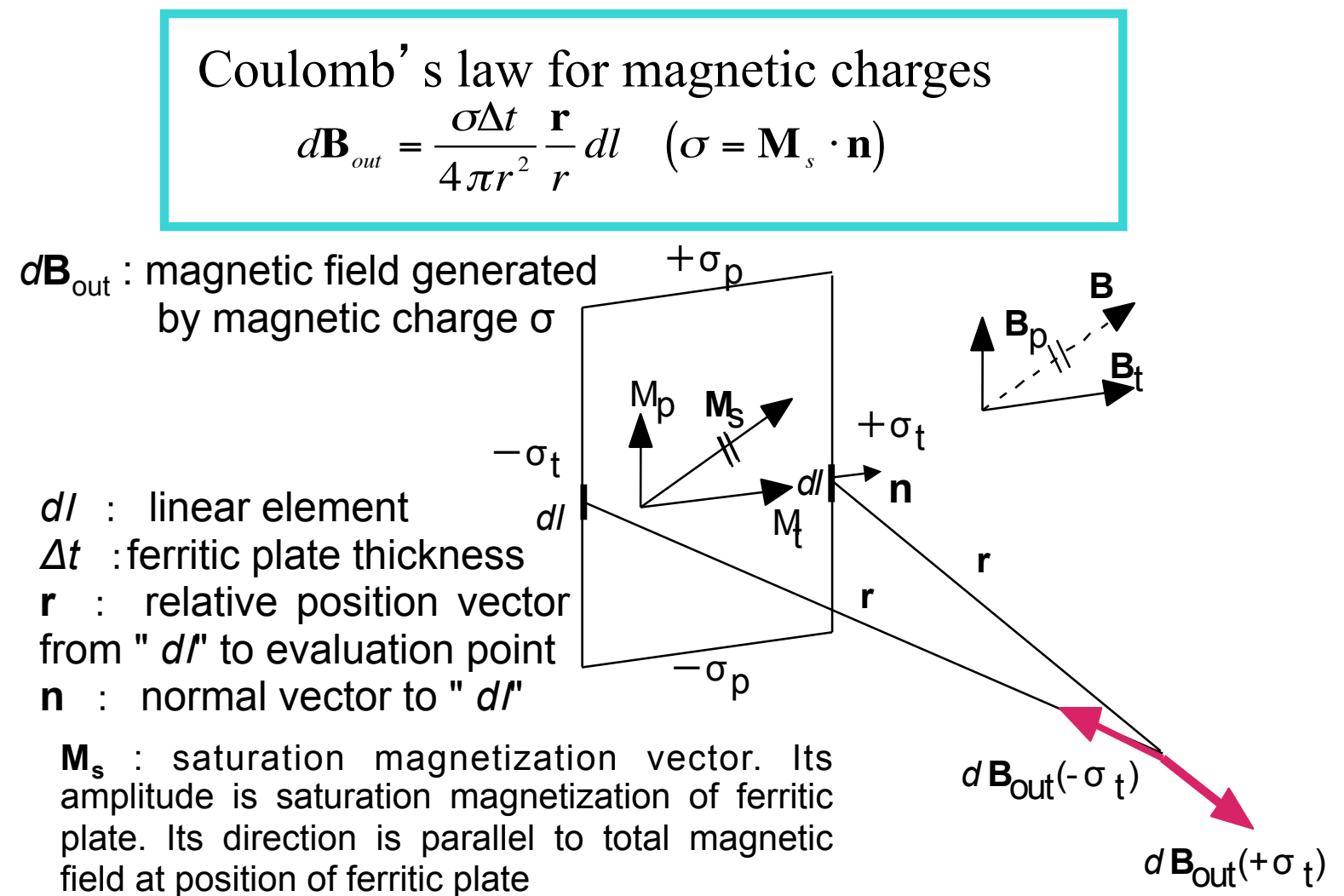




\section{Fig 2}

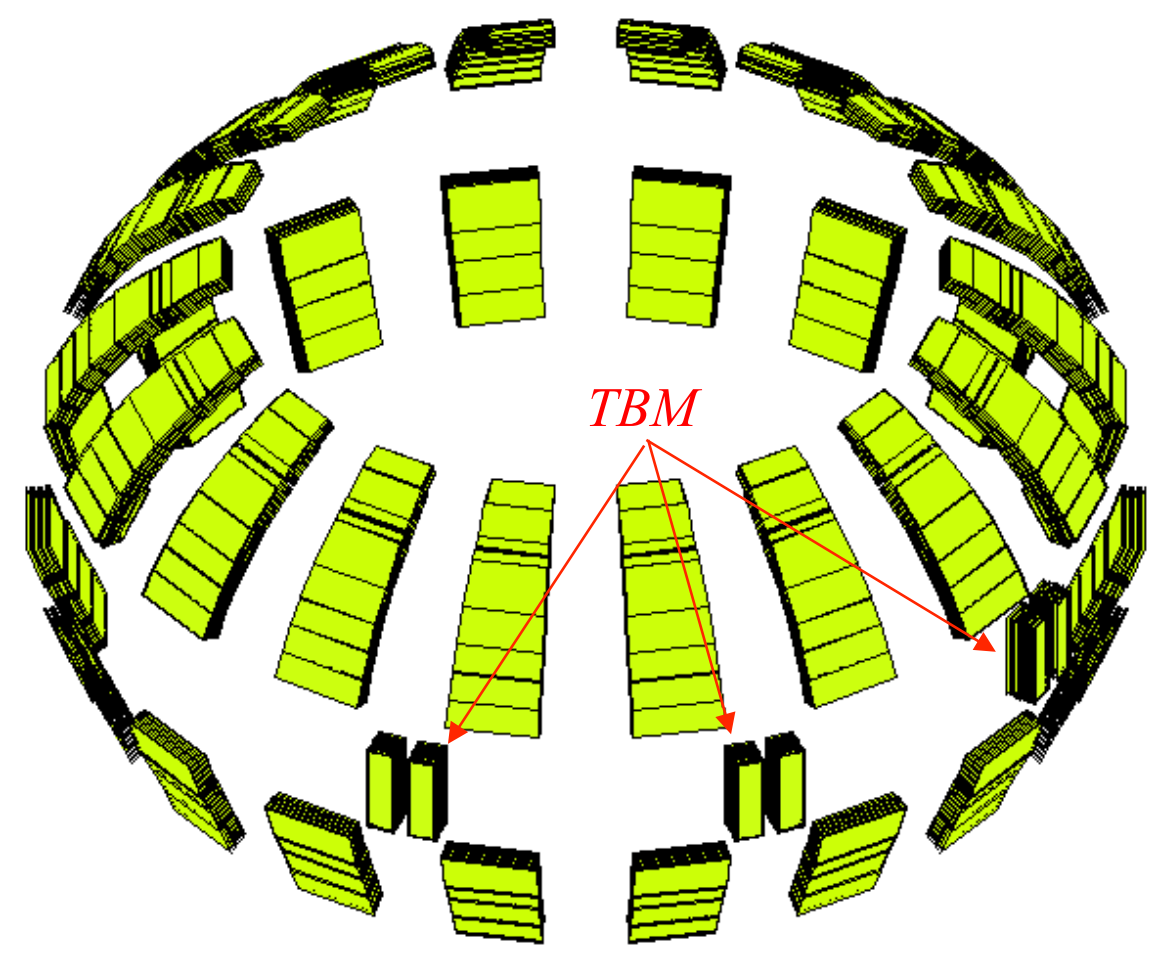

K.Shinohara et.al. 


\section{Fig 3}

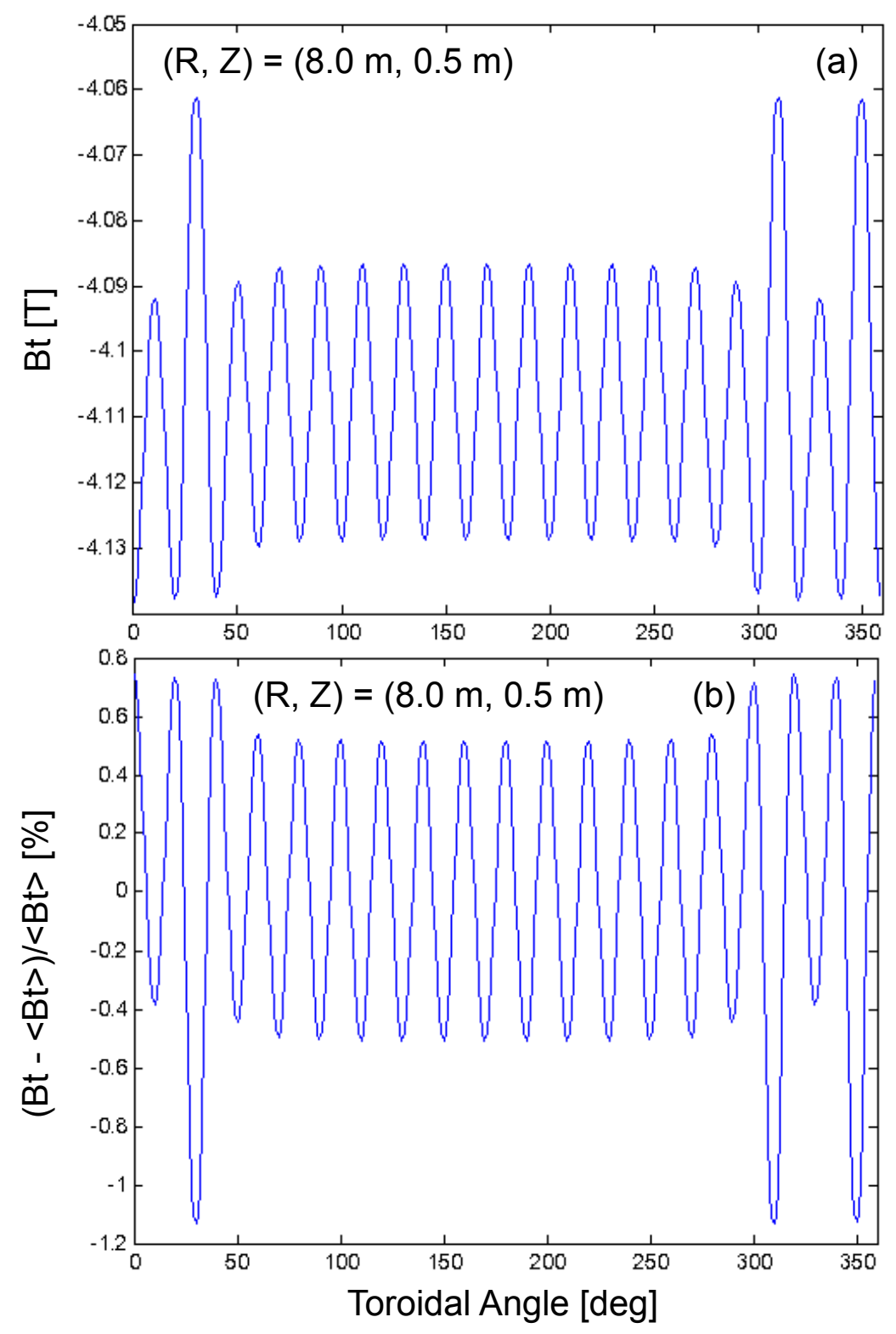




\section{Fig 4}
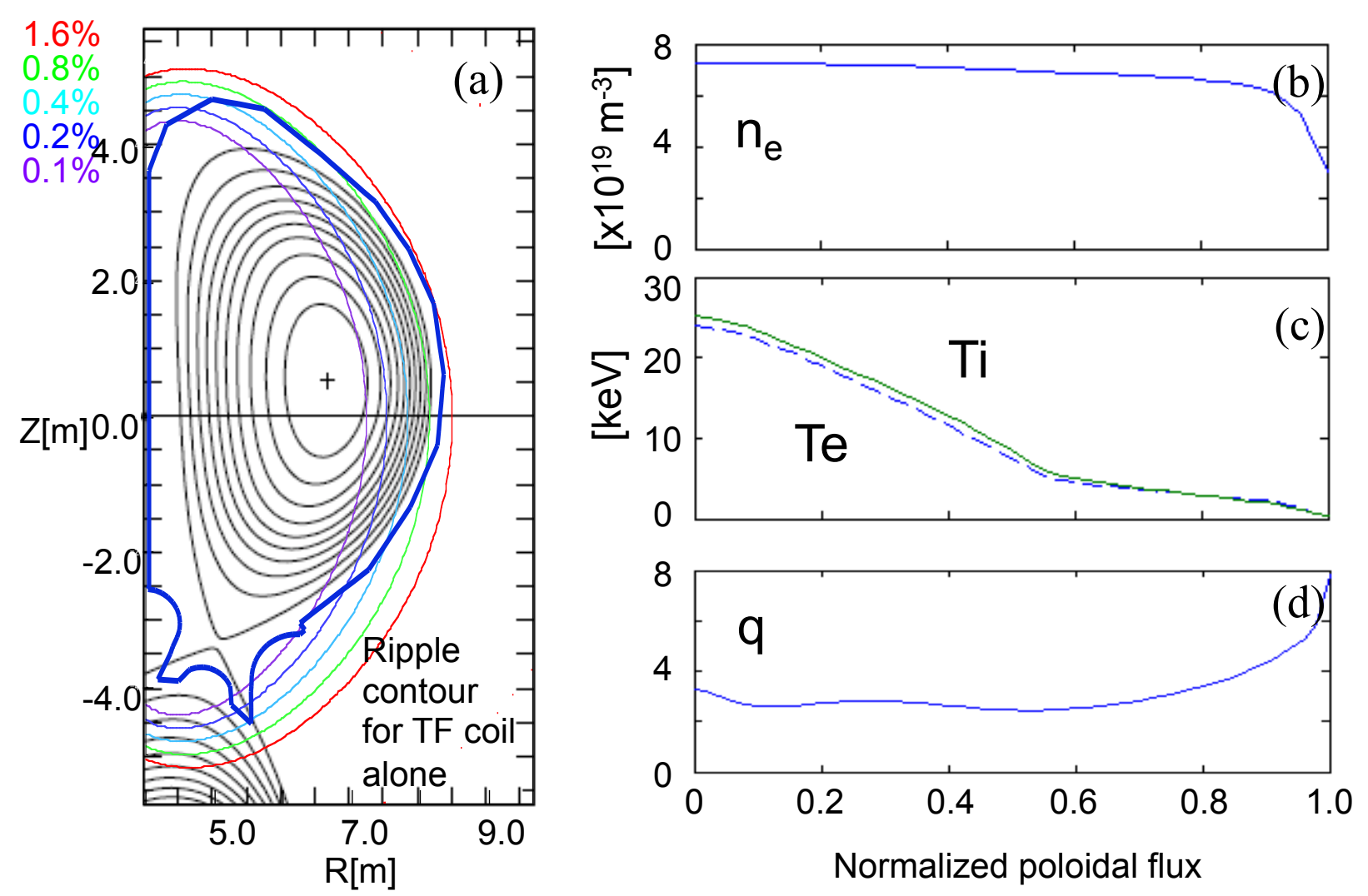


\section{Fig 5}
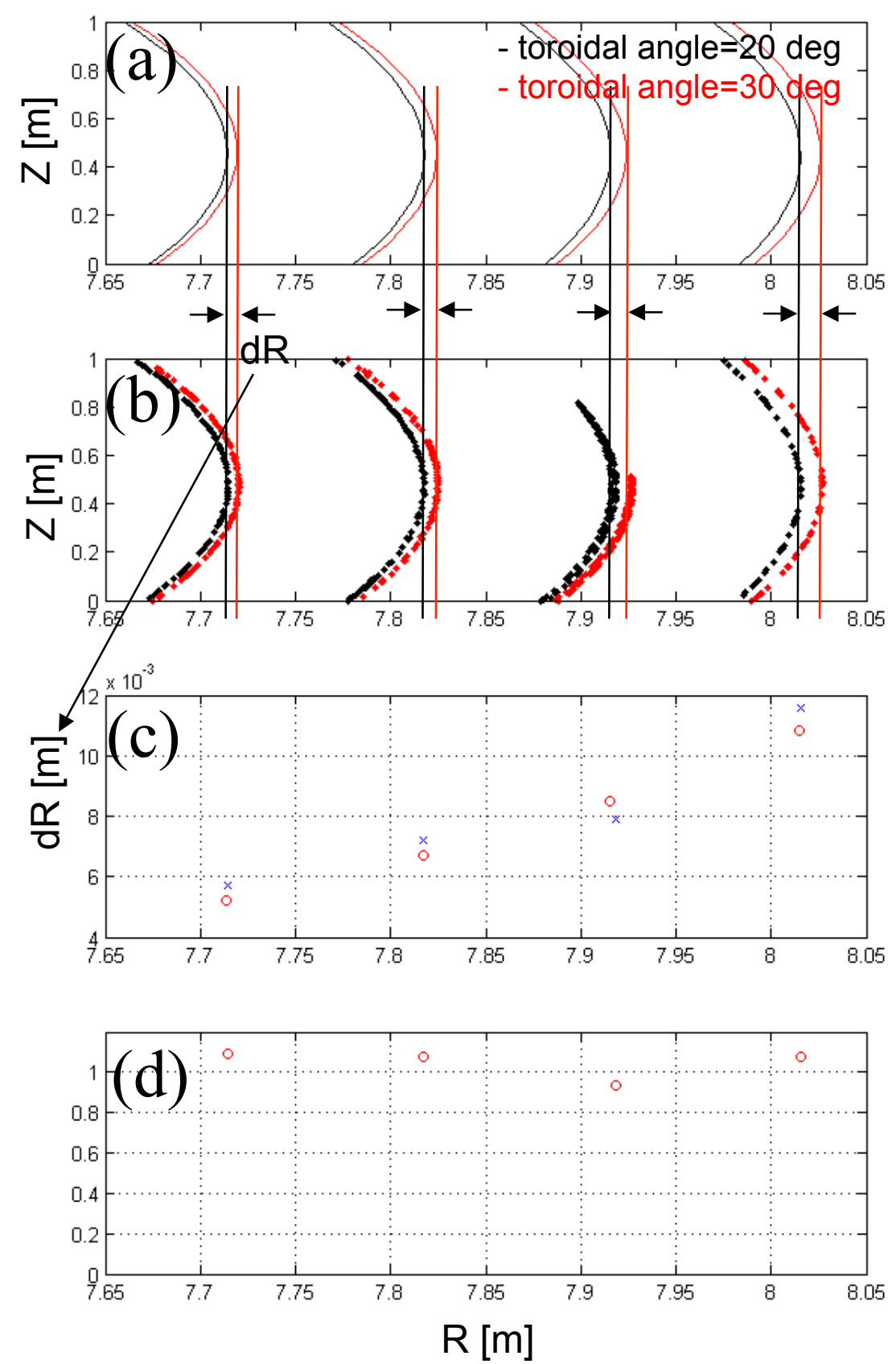


\section{Fig 6}
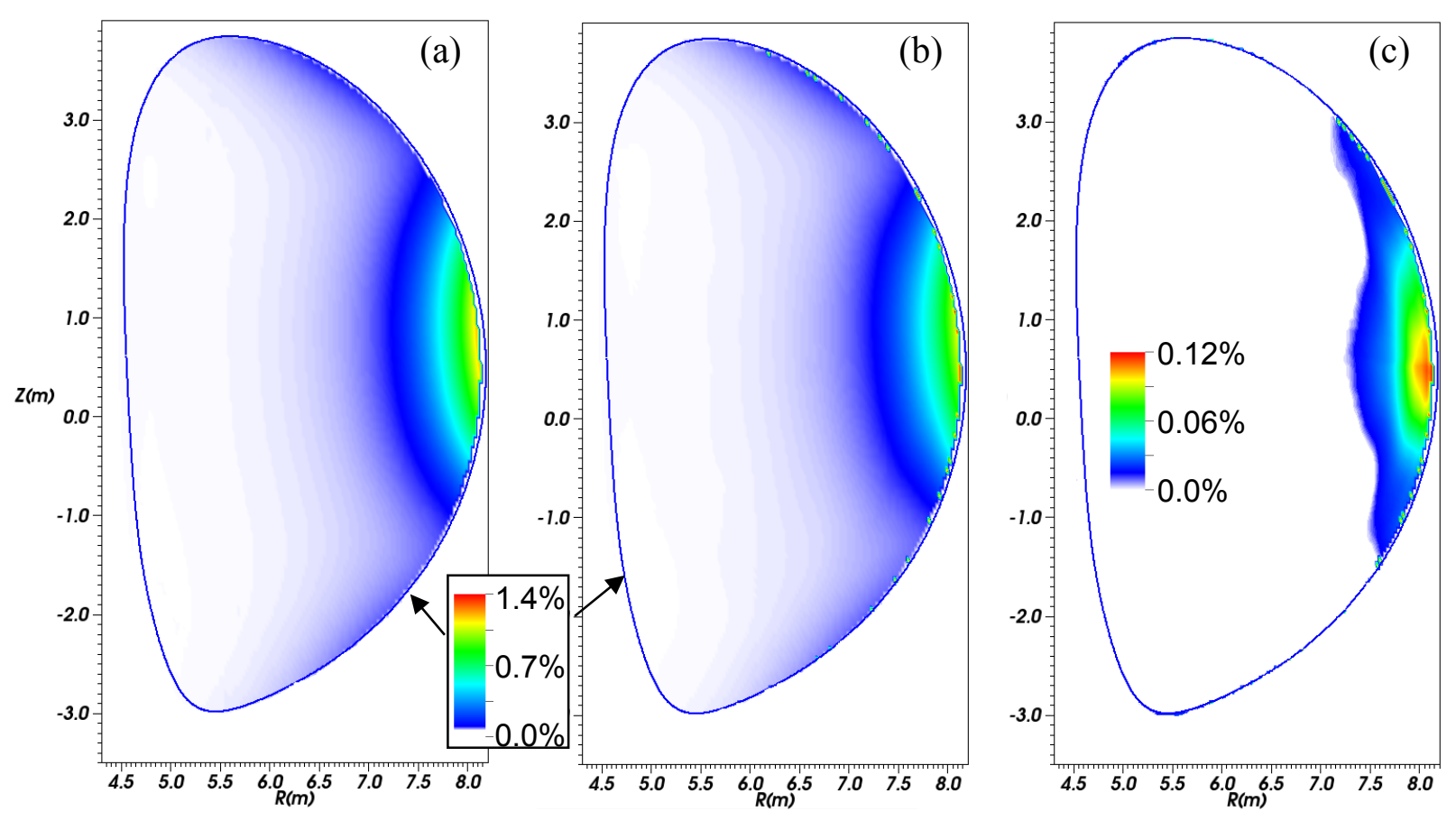


\section{Fig 7}

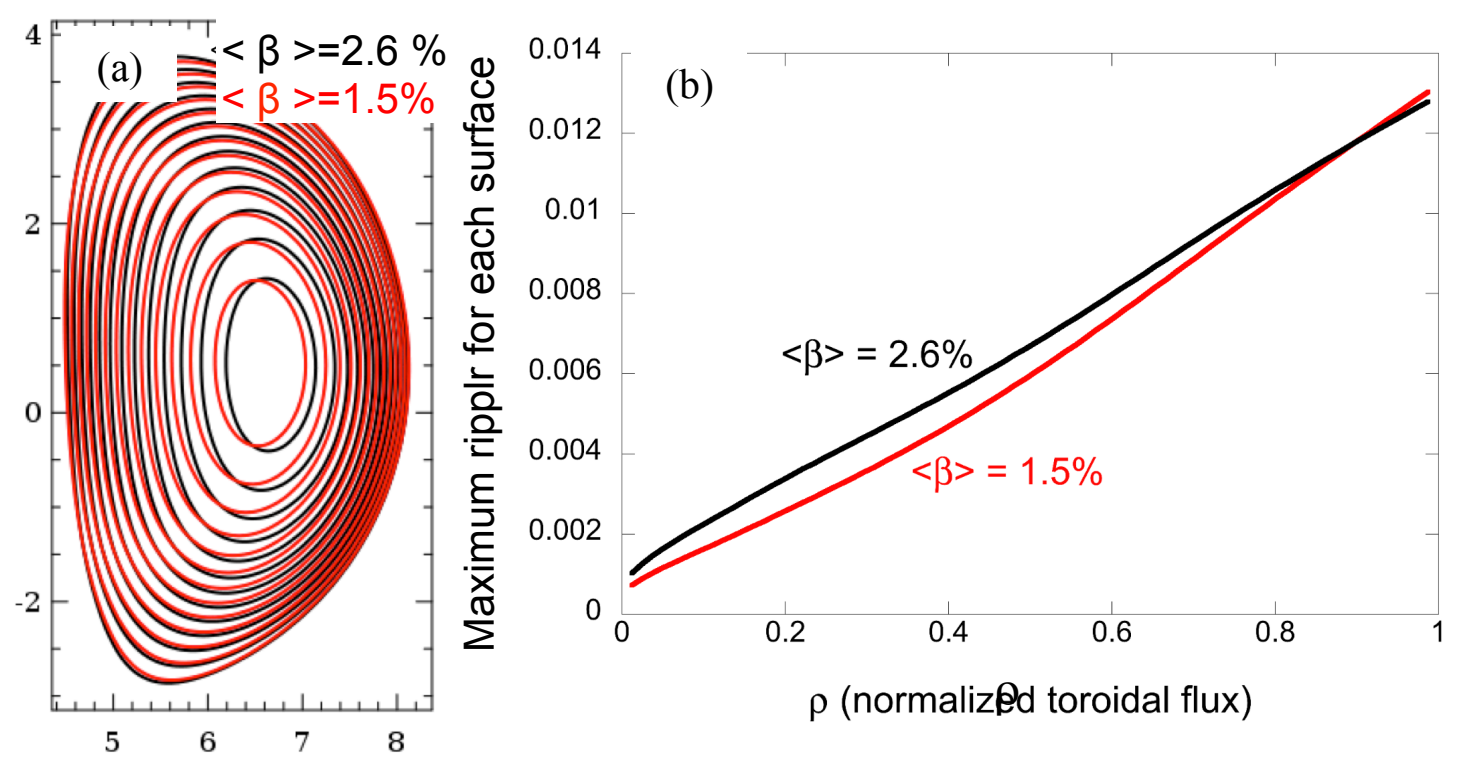




\section{Fig 8}

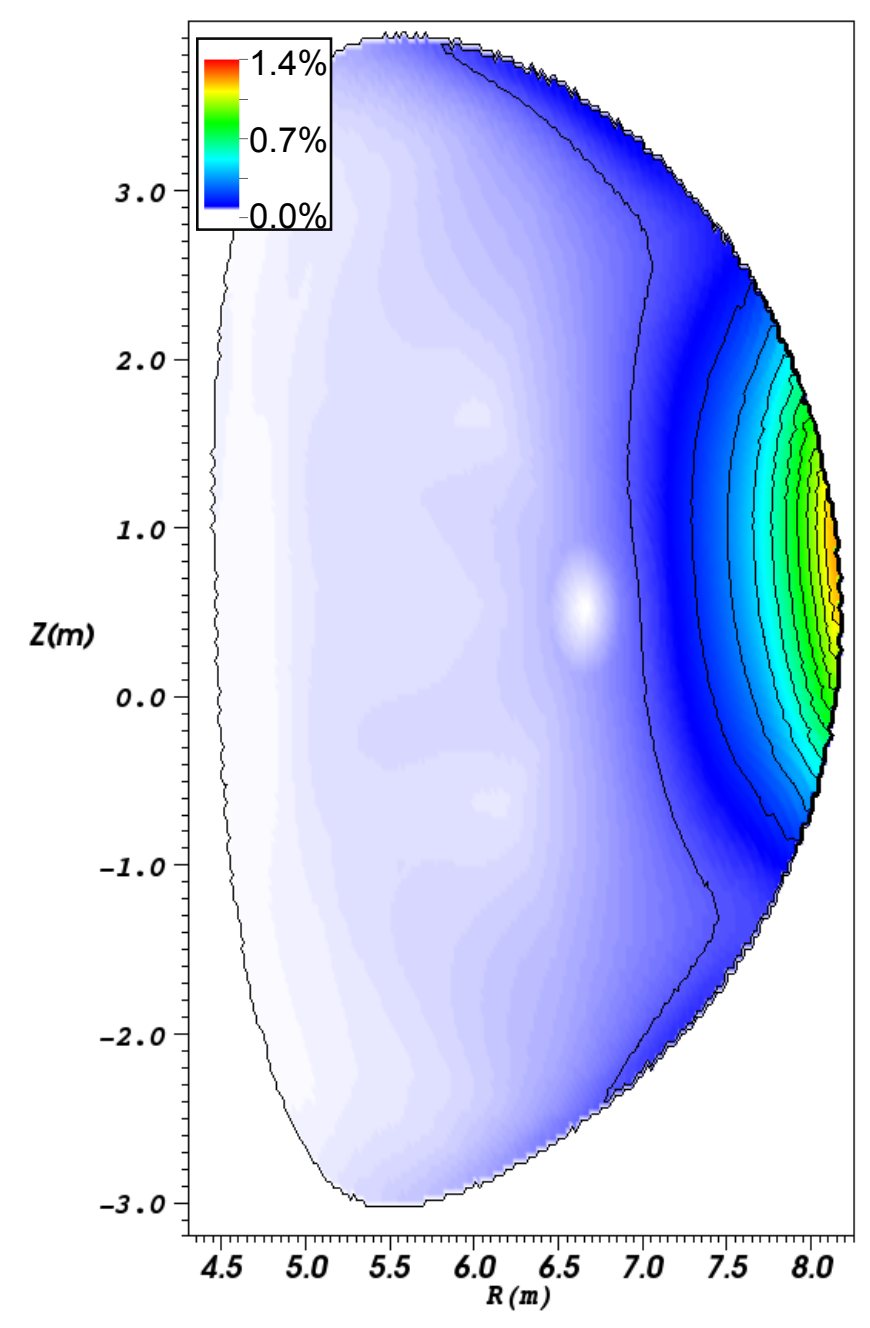

K.Shinohara et.al. 


\section{Fig 9}

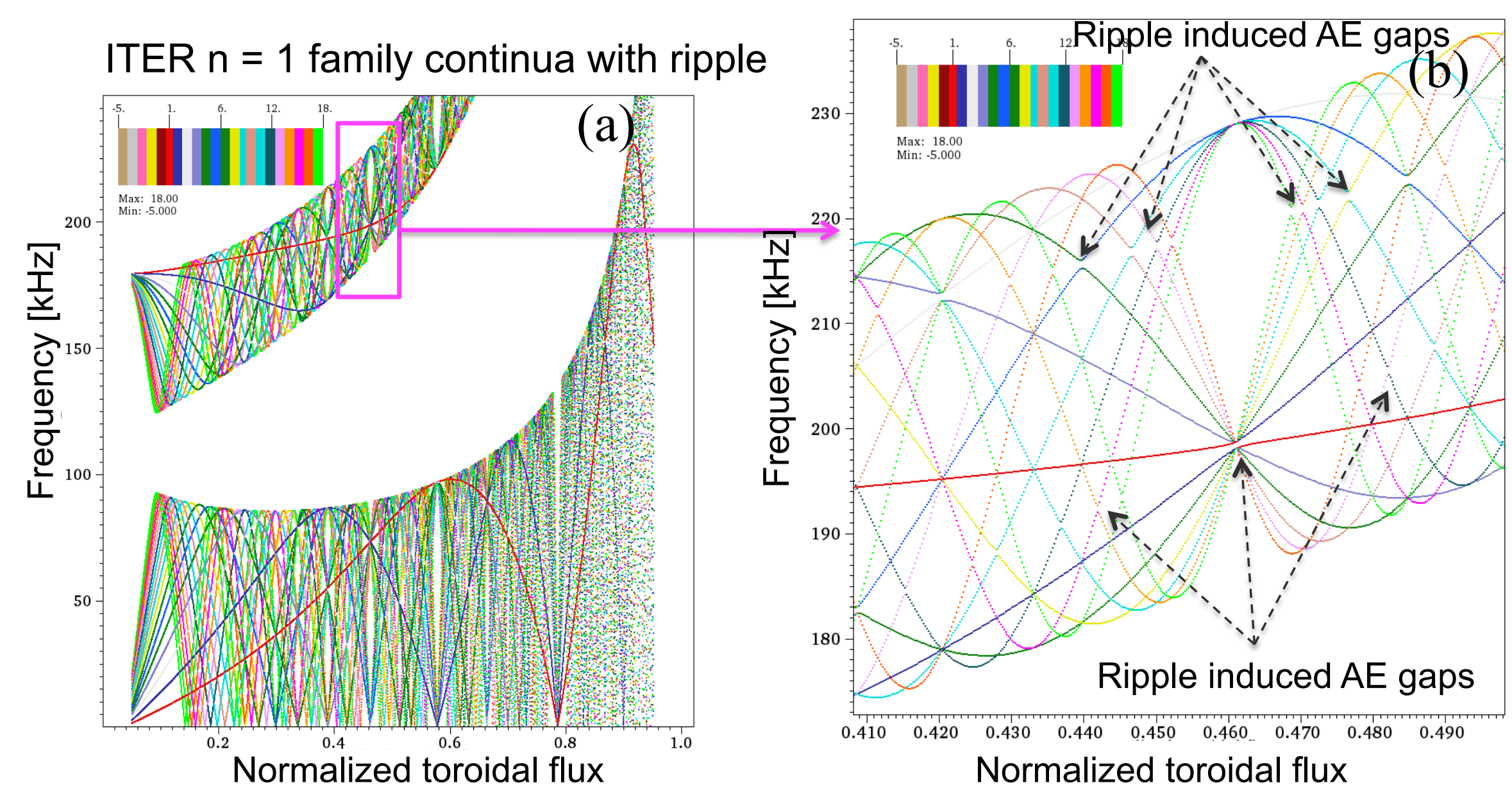

K.Shinohara et.al. 


\section{Fig 10}

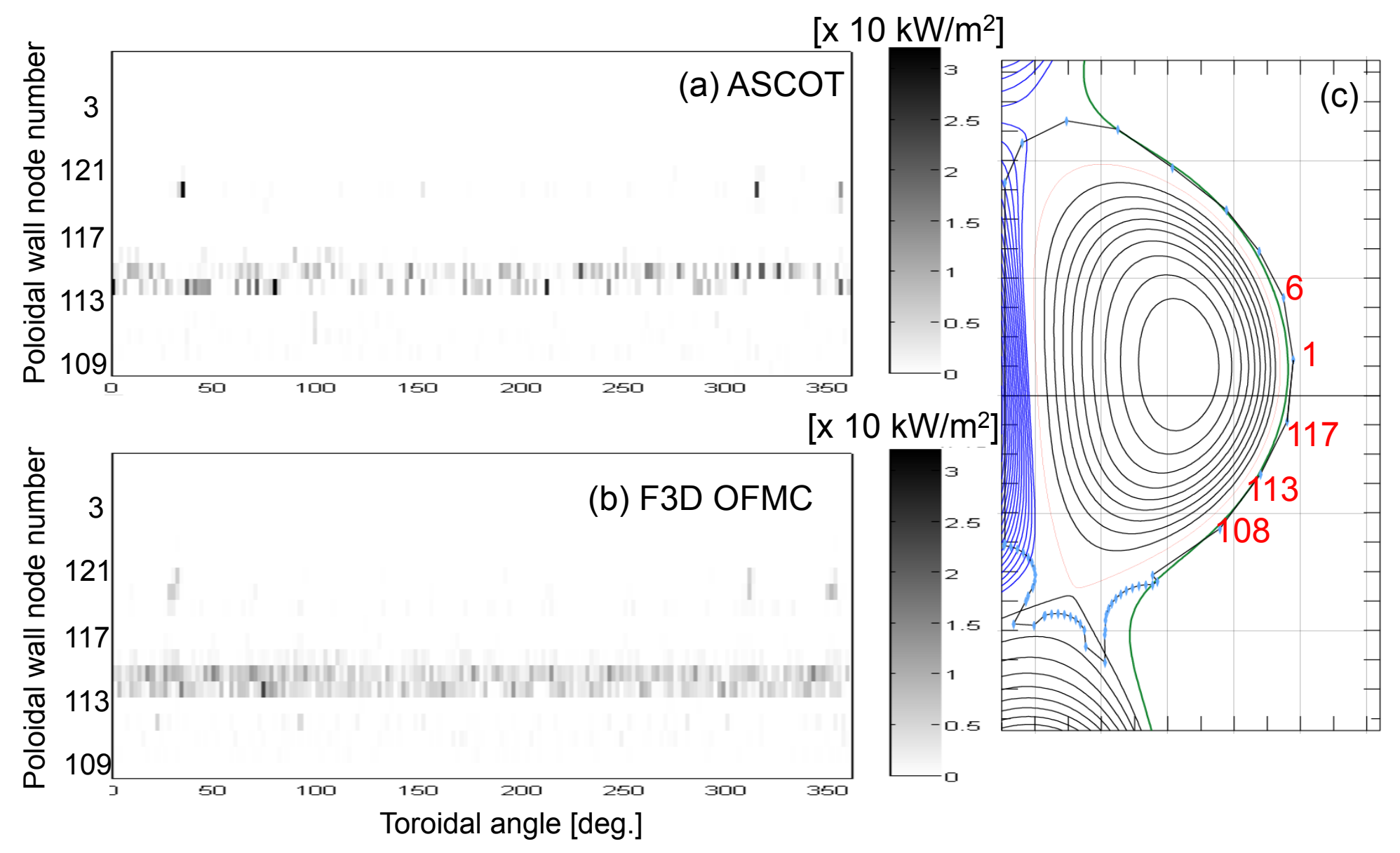




\section{Fig11}
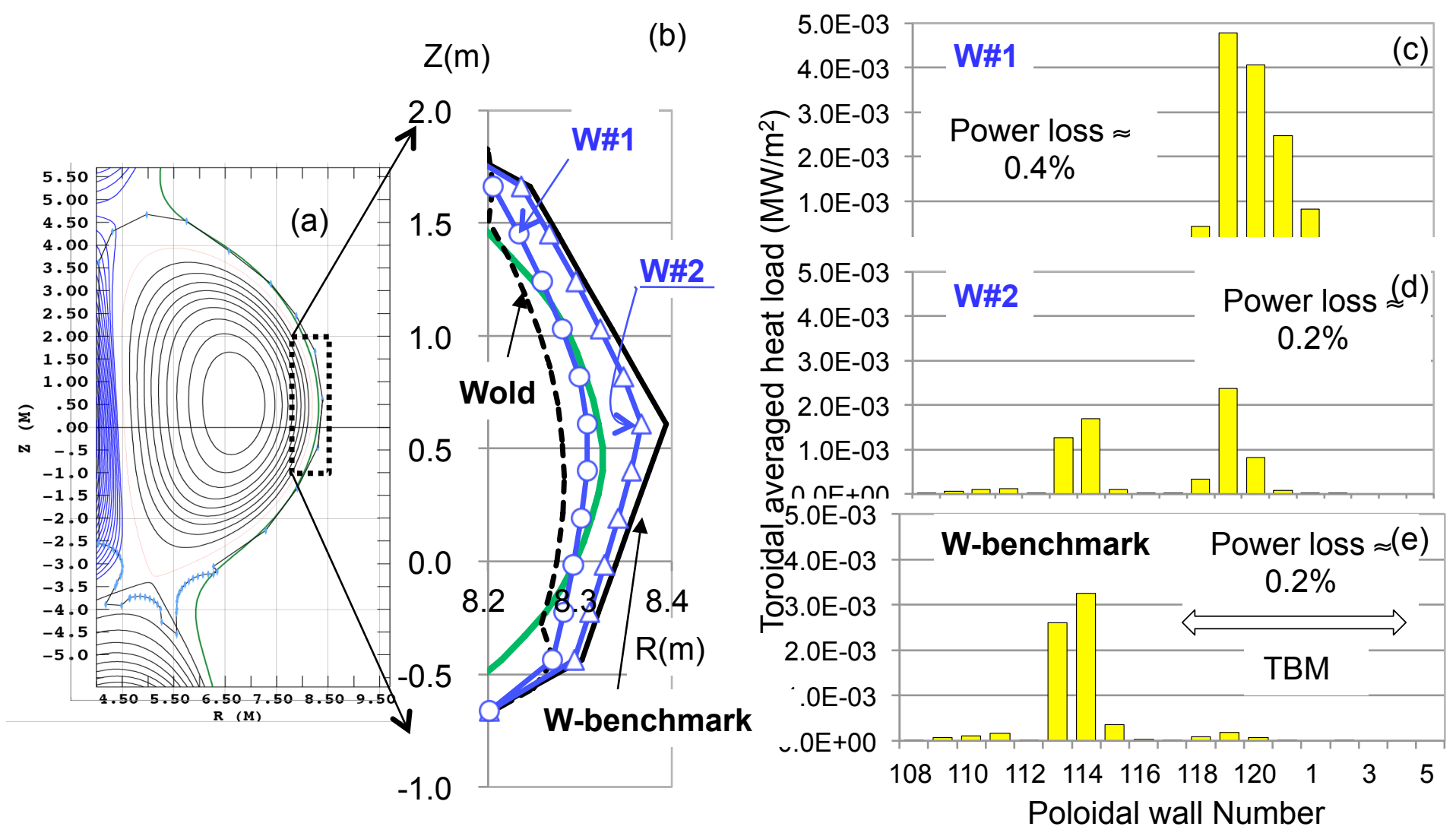

K.Shinohara et.al. 


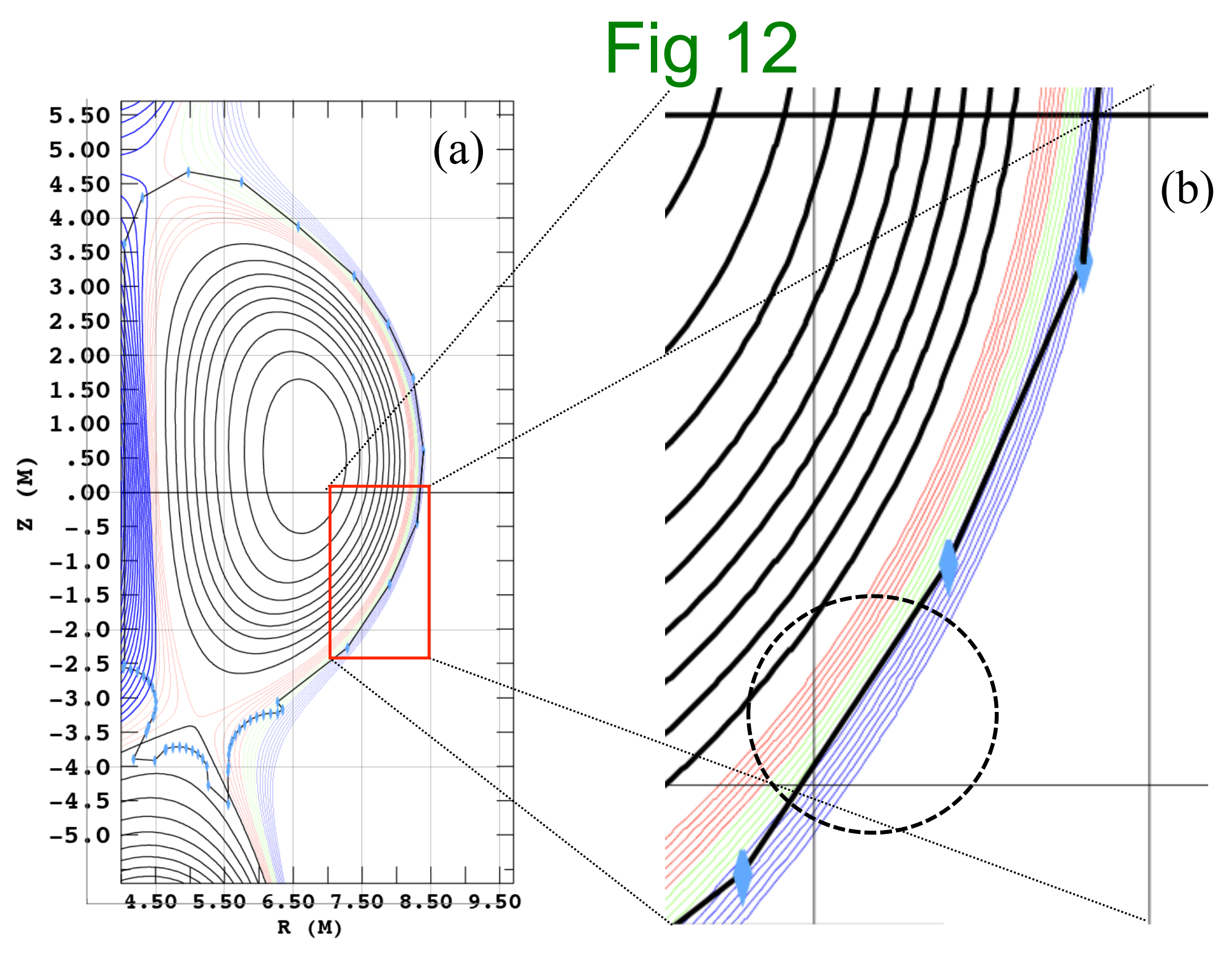

K.Shinohara et.al. 


\section{Fig 13}

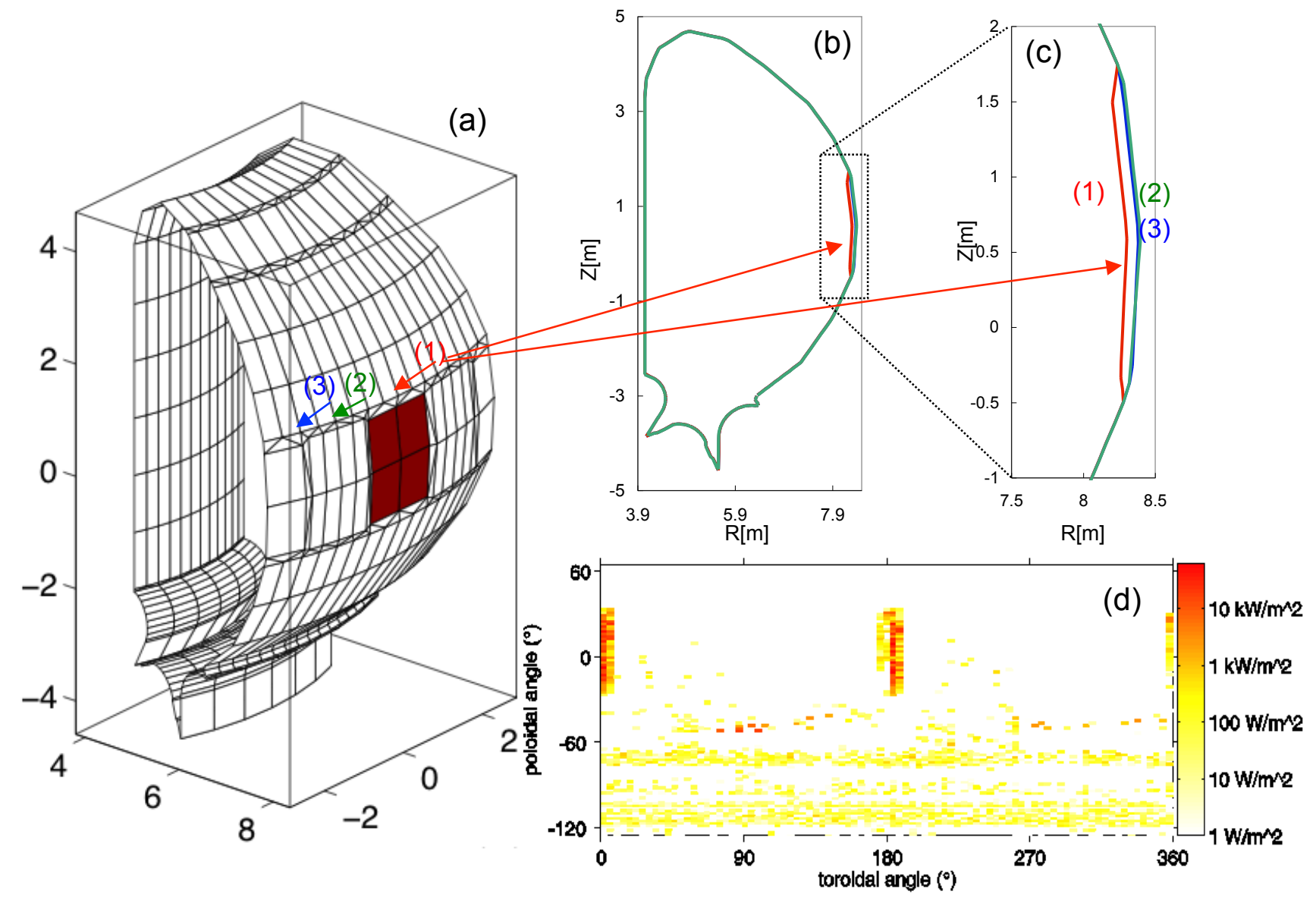




\section{Fig 14}

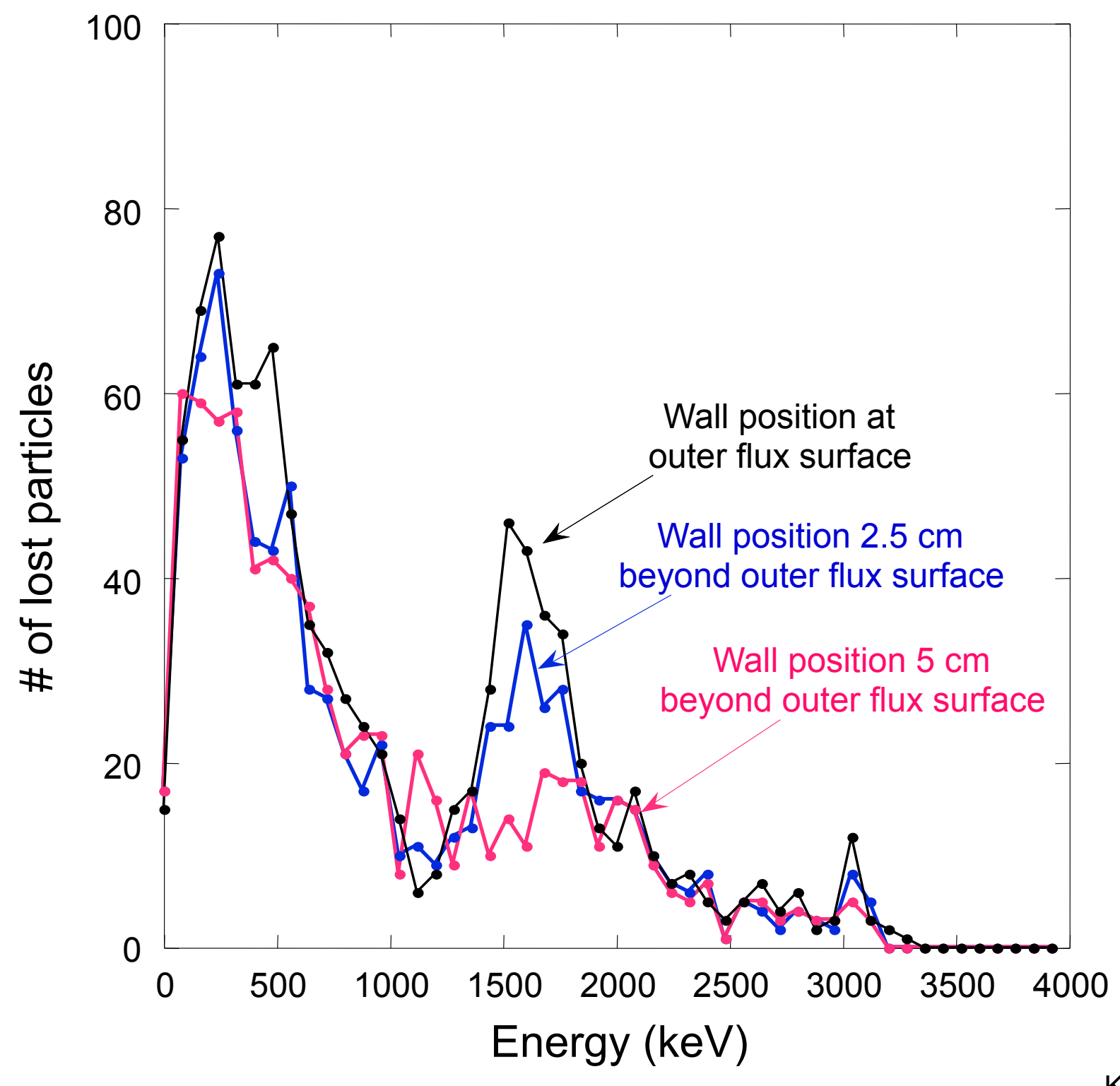

K.Shinohara et.al. 


\section{Fig 15}
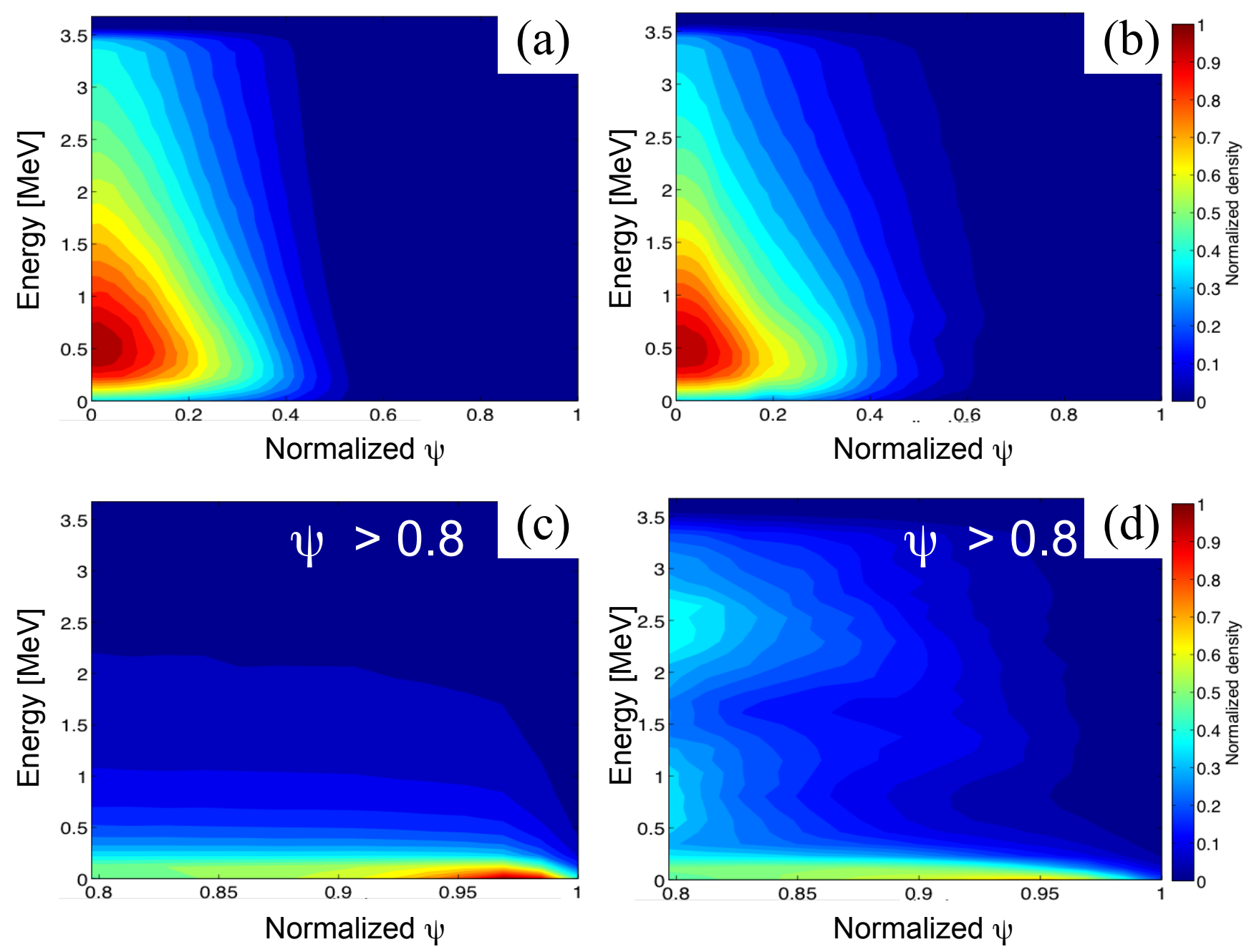


\section{Fig 16}

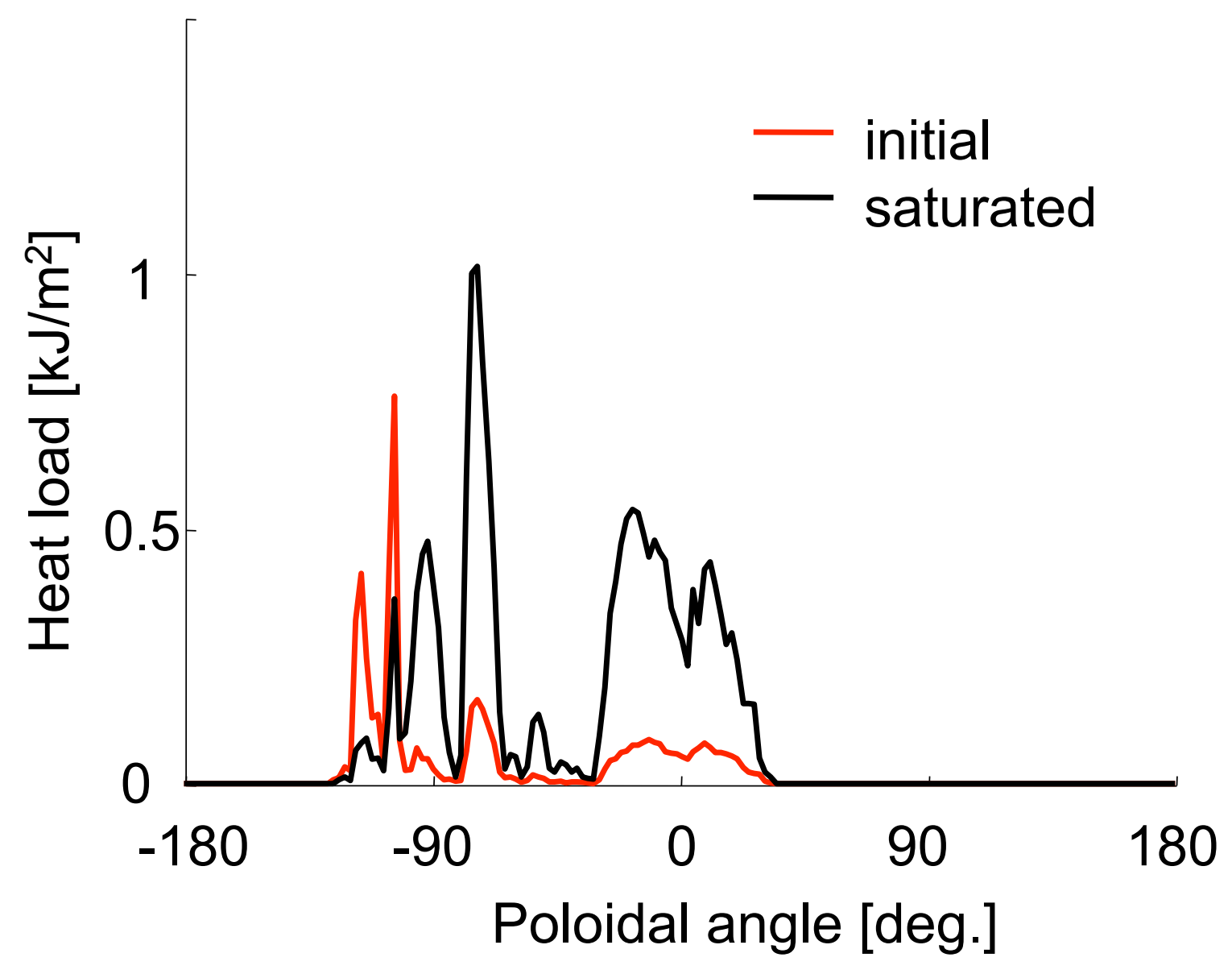




\section{Fig 17}
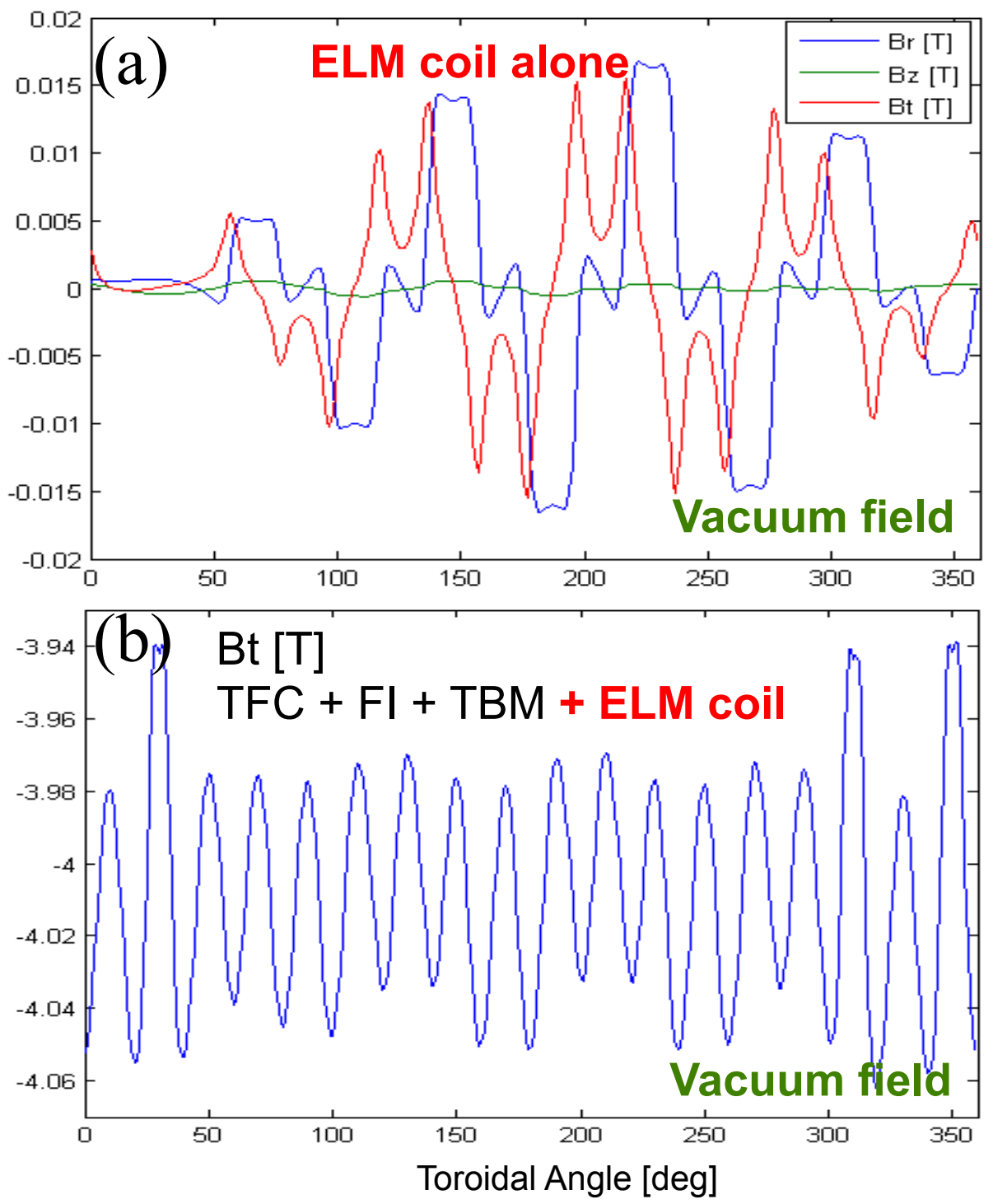